\title{
Lições Aprendidas em uma Iniciativa de Melhoria de Processos de Software sob Diferentes Perspectivas: Membros da Organização, Implementadores e Avaliadores
}

\author{
Gleison Santos, Anne Elise Katsurayama, David Zanetti, Mariano Montoni, \\ Reinaldo C. Silva Filho, Andrea O. S. Barreto, Ana Regina Rocha \\ COPPE/UFRJ - Universidade Federal do Rio de Janeiro \\ Programa de Engenharia de Sistemas e Computação \\ Av. Horácio Macedo, 2030, Prédio do Centro de Tecnologia, Bloco H, Sala 319 \\ Caixa Postal 68511 - CEP 21941-914 - Rio de Janeiro - RJ - Brazil \\ \{gleison, anneelisek, zanetti, mmontoni, cabral, \\ ansoares, darocha\}@cos.ufrj.br
}

\begin{abstract}
COPPE/UFRJ is an MPS.BR Implementation and Appraisal Institution since 2006. In 2008, the Software Quality Area of COPPE's Software Engineering Laboratory was evaluated on the level $E$ of MR-MPS. This experience enabled a huge learning for the team responsible for the implementation and the appraisal of the software processes used by the Software Quality Area as a software development organization. This paper presents a set of lessons learned by COPPE/UFRJ that represents a body of knowledge useful to professionals that act on these three perspectives.
\end{abstract}

Resumo. A Instituição Implementadora e Avaliadora COPPE/UFRJ tem implementado e avaliado o MPS.BR em diversas empresas, acumulando experiências significativas. Em 2008, a Área de Qualidade de Software do Laboratório de Engenharia de Software da COPPE/UFRJ foi avaliada no Nível E do MR-MPS. Esta experiência possibilitou grande aprendizado da equipe responsável pela implementação e avaliação de processos atuando naquele momento como uma organização de desenvolvimento de software. Este artigo apresenta um conjunto de lições aprendidas que constituem um corpo de conhecimento útil a profissionais que atuem sob estas três diferentes perspectivas.

\section{Introdução}

A COPPE/UFRJ é uma Instituição Avaliadora (IA) e Instituição Implementadora (II) MPS credenciada pela SOFTEX desde 2006. A II COPPE tem atuado na implementação de melhoria de processos de software em micro, pequenas, médias e grandes empresas, localizadas no Rio de Janeiro e em outros estados do país, obtendo expressivos resultados nestas implementações. Além disso, a IA COPPE foi responsável por mais de 20 avaliações do MPS.BR, contribuindo para a formação de avaliadores líderes em seu grupo e também para a formação de outros avaliadores através da participação de avaliadores adjuntos de futuras IAs em avaliações MPS lideradas pela COPPE.

As iniciativas de melhoria realizadas em organizações cliente possibilitaram, aos membros da equipe COPPE, a formação de um corpo de conhecimento prático sobre 
melhoria de processos de software. No entanto, por ser uma instituição de pesquisa e desenvolvimento, surgiu a necessidade de estabelecer um ambiente de alta maturidade para possibilitar a aplicação de técnicas de melhoria de processos de software e a aquisição de conhecimentos ainda não tão comuns no mercado, levando em consideração que a maioria das organizações ainda se encontra nos níveis mais baixos de maturidade do MR-MPS. Por isso, no final de 2007, os membros da II e da IA COPPE decidiram pela implementação do nível E do MR-MPS versão 1.2 [SOFTEX 2007] na Área de Qualidade do Laboratório de Engenharia de Software (LENS) da COPPE/UFRJ. A implementação de melhoria de processos no LENS visa à construção de um ambiente de alta maturidade que possa ser utilizado como laboratório para a aplicação de estratégias de implementação de melhoria de processos de software, e para que estas estratégias sejam utilizadas em organizações cliente, aumentando a probabilidade de sucesso de iniciativas de melhoria. Este desafio trouxe aos implementadores uma experiência única, pois de consultores passaram ao papel de colaboradores e foram avaliados. Além disso, muitas lições aprendidas foram obtidas a partir desta experiência.

O objetivo deste artigo não é discutir detalhes de como a implementação dos processos foi conduzida, mas apresentar um conjunto de lições aprendidas a partir da implementação do Nível E do MR-MPS sob as perspectivas de uma organização que está implantando um programa de melhoria de processos de software, de membros de uma instituição implementadora responsável pela condução da iniciativa de melhoria de processos de software e de avaliadores do MPS.BR sendo eles próprios os avaliados. A próxima seção discute sobre a implementação de iniciativas de melhoria de processos de software. Na seção 3 é apresentada a estrutura da Área de Qualidade de Software do LENS, unidade organizacional avaliada no nível E do MR-MPS versão 1.2. Na seção 4 são apresentadas as lições aprendidas a partir desta experiência. E, por fim, na seção 5 são apresentadas as conclusões e perspectivas futuras deste trabalho.

\section{Implementação de Iniciativas de Melhoria de Processos de Software}

O trabalho em processo de software apóia a construção da qualidade no software ao manter o foco em como o software é construído e avaliado. Infelizmente, produtos de software são geralmente desenvolvidos utilizando processos ad hoc que geralmente residem na cabeça daqueles que os executam e supervisionam. Como consequiência, projetos de software são geralmente mal coordenados, supervisionados inadequadamente e falham em entregar produtos que demonstrem grande qualidade. Tornar o processo de desenvolver software explícito pode ajudar a resolver muitos destes problemas e, de fato, parece que o aumento do rigor e detalhe das definições de processo de software leva a um aumento na eficiência no desenvolvimento de produtos de software cuja qualidade é tanto assegurada como demonstrável [Osterweil 1996].

Modelos de Capacidade e Maturidade têm foco na melhoria de processos numa organização. Eles contêm os elementos essenciais de processos para uma ou mais disciplinas e descrevem um caminho evolucionário de melhoria partindo de processos ad hoc e imaturos até chegar a processos disciplinados e maduros com a melhora da qualidade e eficiência [Chrissis et al. 2006]. BOEHM [2006] diz que ao longo do tempo, muitas empresas têm relatado bom retorno de investimento devido à redução de retrabalho ao adotarem um modelo de maturidade, isso pode ser comprovado, por exemplo, em [Ferreira et al. 2007]. 
O MPS.BR [SOFTEX 2007] é um programa para Melhoria de Processo do Software Brasileiro coordenado pela Associação para Promoção da Excelência do Software Brasileiro (SOFTEX) e tem como objetivo definir um modelo de melhoria e avaliação de processo de software, preferencialmente para as micro, pequenas e médias empresas, de forma a atender as suas necessidades de negócio e a ser reconhecido nacional e internacionalmente como um modelo aplicável à indústria de software. Este é o motivo pelo qual ele está aderente a modelos, como o CMMI (Capability Maturity Model Integration) [Chrissis et al. 2006], e normas internacionais, como a ISO/IEC 12207 [ISO/IEC 2008] e a ISO/IEC 15504 [ISO/IEC 2003]. O MPS.BR também define regras para sua implementação e avaliação, dando sustentação e garantia de que está sendo empregado de forma coerente com as suas definições. O Nível E do MR-MPS (Modelo de Referência do MPS.BR) engloba a implementação dos processos Gerência de Requisitos, Gerência de Projeto, Medição, Garantia da Qualidade, Gerência de Configuração, Aquisição, Gerência de Reutilização, Gerência de Recursos Humanos, Definição do Processo Organizacional, Avaliação e Melhoria do Processo Organizacional.

\section{A Área de Qualidade de Software do LENS}

A COPPE/UFRJ é formada por um conjunto de programas de pós-graduação, entre eles o Programa de Engenharia de Sistemas e Computação (PESC). Cada programa é formado por áreas de pesquisa e laboratórios. A área de Engenharia de Software faz parte do PESC e é responsável por atividades de ensino, pesquisa, consultoria e, também, pelo Laboratório de Engenharia de Software (LENS). O LENS, por sua vez, está organizado em três áreas: Qualidade de Software, Engenharia de Software Experimental e Reutilização.

A unidade organizacional avaliada no nível E do MR-MPS versão 1.2 foi a área de Qualidade de Software do LENS, que, atualmente é composta pela maior parte da equipe de implementadores e avaliadores da COPPE/UFRJ. A área de Qualidade de Software possui um portfólio de projetos que engloba customizações do ambiente de gestão do conhecimento CORE-KM [Galotta et al. 2004] para clientes externos; evolução do ambiente de desenvolvimento de software TABA [Montoni et al. 2006], que é utilizado por organizações para apoiar iniciativas de melhoria; e desenvolvimento dos produtos de software que fazem parte de dissertações de mestrado e teses de doutorado desenvolvidas na área de qualidade do LENS. Estes três tipos de produtos fizeram parte do escopo da avaliação no nível E do MR-MPS.

No início das atividades para implementação do nível E do MR-MPS, muitas das práticas previstas já eram utilizadas. Além disso, os envolvidos no programa de melhoria tinham experiência em implementação de processos, tanto com o modelo MPS.BR [SOFTEX 2007] quanto com o modelo CMMI [Chrissis et al. 2006]. A equipe do LENS também possuía experiência na liderança de avaliações MPS.BR e na participação em equipes de avaliação SCAMPI. Os esforços para a implementação do nível E do MR-MPS foram iniciados em Outubro de 2007 e a avaliação foi concluída com sucesso em maio de 2008. A estratégia utilizada para a implementação do nível E do MRMPS na área de Qualidade do LENS foi a mesma utilizada pela equipe de consultoria da COPPE/UFRJ nas iniciativas de melhoria: o SPI-KM [Santos et al. 2007b]. Lições aprendidas no contexto do SPI-KM [Santos et al. 2007a] também foram aplicadas na condução da implementação de melhoria de processos. 


\section{Lições Aprendidas na Implementação do Nível E do MR-MPS no Labora- tório de Engenharia de Software da COPPE/UFRJ}

Visto que a COPPE/UFRJ possui um quadro de implementadores com vasta experiência em implementações de diversos níveis do MR-MPS e também do CMMI, não sofre pressões mercadológicas e possui total apoio da alta gerência para a implementação, pode-se dizer que o quadro era totalmente favorável ao sucesso. Mesmo assim, durante a implementação do nível E do MR-MPS, a equipe enfrentou diversos desafios. Alguns deles comuns às organizações que atuam na indústria de software e outros não tão comuns, visto que a COPPE/UFRJ é uma instituição de pesquisa e desenvolvimento sem fins lucrativos. A transposição desses desafios para a conquista do nível $\mathrm{E}$ fez com que a equipe identificasse uma série de lições aprendidas através de relatos da equipe envolvida diretamente com a implementação dos processos e serão descritos a seguir.

Para melhor entendimento da importância e significado das lições aprendidas com esta avaliação, alguns fatos devem ser registrados:

- Para maior segurança, havia dois representantes da unidade organizacional na equipe de avaliação possibilitando que nas duas mini-equipes, a serem formadas durante as avaliações inicial e final, houvesse um representante da COPPE. Estes representantes eram avaliadores MPS (um avaliador líder inicial e o outro um avaliador adjunto que já tinha participado de muitas avaliações).

- O patrocinador é implementador e avaliador líder experiente MPS. Os gerentes de projeto e os responsáveis por Medição, Gerência de Configuração, Qualidade, Processos, Gerência de Recursos Humanos e Gerência de Reutilização são todos implementadores MPS, com experiência em muitas empresas já avaliadas. Todos menos um, também, são avaliadores MPS.

- Não houve um único item marcado como requerido na avaliação inicial e muito poucos itens foram assinalados como oportunidades para futuras melhorias.

\subsection{Lições Aprendidas na Perspectiva da Organização sendo Avaliada}

Mesmo com o toda a experiência prévia, a implementação do nível E não foi simples. A COPPE/UFRJ, assim como qualquer outra organização que busque a melhoria de seus processos, enfrentou diversos desafios. Alguns desses desafios foram determinantes para a identificação das lições aprendidas a seguir.

É preciso planejamento adequado dos prazos previstos da iniciativa de melhoria de processos de software de forma a atendê-los com segurança. Um dos maiores desafios na implantação do Nível E foi relacionado aos prazos. Tratava-se de um grupo heterogêneo que tinha de lidar com diversas responsabilidades de focos distintos. Por se tratarem de alunos de mestrado e doutorado, todos necessitavam dedicar tempo para suas dissertações e teses, o que ocupava um tempo considerável e que era tratado com a mesma prioridade que qualquer outra atividade. Além disso, a equipe dos projetos era a mesma equipe responsável pelos projetos de melhoria em empresas clientes. Sendo assim, quase todo o tempo disponível era dedicado a essas duas atividades. Esses fatores fizeram com que o planejamento dos projetos levasse em consideração as alocações e disponibilidades de cada membro da equipe, e encontrar disponibilidade para os recursos não foi uma tarefa simples neste cenário. 
Os recursos alocados à iniciativa de melhoria de processos de software são limitados e precisam ser usados sabiamente. Por se tratar de uma instituição de ensino e pesquisa, a COPPE/UFRJ não possui fins lucrativos. Também não foi disponibilizado qualquer apoio de agência de fomento e se dispunha, apenas, daqueles contratados por desenvolvimentos externos e consultorias em melhoria de processos de software. Portanto, era necessário compatibilizar os recursos disponíveis com os recursos requeridos para institucionalização dos processos.

O esforço associado a uma iniciativa de melhoria de processos de software é grande e devido a isso é preciso dimensionar e priorizar as atividades para se atingir o sucesso. Mesmo com todo um cenário tecnicamente favorável, com profissionais altamente qualificados, a implementação exigiu um grande esforço da equipe, ou seja, o conhecimento técnico da equipe auxiliou no atendimento completo dos processos do nível, mas não diminuiu o trabalho para implementá-los. Como toda a equipe participava ativamente da implementação de todos os processos do MR-MPS, era necessário dimensionar as atividades a serem realizadas de forma adequada e também estabelecer prioridades para as pendências, de forma que uma pendência sob responsabilidade de um membro da equipe não impactasse outra pendência sob responsabilidade de outro membro. É possível que uma redefinição dos processos com foco na otimização dos mesmos diminua o esforço associado com a sua execução.

Deve-se procurar gerenciar a diversidade de idéias de forma a diminuir as divergências entre a equipe e concentrar esforços em soluções não conflitantes. Apesar de ser também um ponto favorável à implementação, a diversidade de idéias do grupo poderia gerar divergência de opiniões e falta de objetividade na escolha das diversas soluções possíveis para cada aspecto requerido pelo modelo. Era necessário homogeneizar o conhecimento inerente aos processos a todos os membros da unidade organizacional e gerenciar as sugestões de melhoria de forma a garantir o atendimento dos prazos.

Soluções não ideais terão que ser implementadas e, portanto, deve-se procurar aceitá-las devido às restrições inerentes à condução da iniciativa de melhoria de processos de software. Devido às pressões de prazo e aos recursos limitados, a equipe teve de aprender a se contentar com soluções possíveis na ausência de soluções ótimas exeqüíveis, visto que era um grupo com profundo conhecimento especializado e que desejava realizar uma implementação o mais próxima possível da ideal.

Deve haver uma divisão clara de responsabilidades para diminuir as chances de conflito entre a equipe, minimização de esforço e garantir que todas as tarefas necessárias serão realizadas. A implementação dos processos aderentes ao nível E do MR-MPS exigiu a divisão bem definidas de responsabilidades entre os membros da equipe, mesmo que estes se sentissem aptos e motivados para realizar qualquer das tarefas. Estabelecer responsáveis para cada processo aumenta o comprometimento e facilita a identificação precoce de problemas, desde que seja feita uma monitoração periódica da situação dos processos.

Os benefícios da iniciativa de melhoria de processos de software devem ser reconhecidos para aumentar o comprometimento com as ações realizadas. Durante a implementação, a equipe sentiu claramente a necessidade de um mecanismo de reconhecimento dos benefícios associados aos esforços de melhoria executados, de forma que estas melhorias fossem percebidas mais diretamente. Mesmo estando suficientemente 
comprometidos com a melhoria, o reconhecimento dos benefícios serviria para aumentar ainda mais este comprometimento e também para motivar os membros em busca da melhoria contínua através dos níveis superiores de maturidade.

Todo o esforço deve ser de alguma forma recompensado. Por mais que haja comprometimento, é necessário recompensar aqueles que se dedicam intensamente na obtenção de um objetivo. Todos necessitam sentir-se recompensados pelos esforços prestados. A manutenção da satisfação da equipe é fundamental para manter ou aumentar o comprometimento da equipe. É muito importante pesquisar e utilizar as diversas formas para recompensar aqueles que se dedicam em busca de um ideal coletivo.

\subsection{Lições Aprendidas na Perspectiva da Instituição Implementadora}

Apesar de possuir um quadro favorável a iniciativa de melhoria, pois a maior parte dos membros da unidade organizacional avaliada são implementadores e alguns são avaliadores MPS.BR credenciados pela SOFTEX, além de possuírem larga experiência em melhoria de processos, o fato de atuar ao mesmo tempo como organização avaliada e responsável pela implantação do programa de melhoria também trouxe importantes lições aprendidas apresentadas a seguir.

$O$ trabalho em equipe e em grupos grandes é fundamental para o sucesso da iniciativa de melhoria de processos. Nas implementações em organizações clientes, a COPPE/UFRJ geralmente trabalha com equipes menores, constituídas por, em média, dois consultores por cliente. Era necessário desenvolver uma sinergia entre os membros da equipe como um todo e também estabelecer um sincronismo entre as atividades das equipes de consultoria e a equipe de implementação do Nível E.

Sem uma equipe totalmente dedicada e com os conhecimentos necessários é bastante difícil implantar uma melhoria de processos da magnitude da melhoria requerida para o nível E do MR-MPS em um período tão curto. Todos estavam comprometidos com um só objetivo. A dedicação e a ajuda mútua de todos os envolvidos, além dos conhecimentos e das experiências adquiridas nas implementações, avaliações e cursos internos e externos realizados pela equipe foi um fator crucial para o sucesso.

A manutenção do foco nas responsabilidades de cada membro, de acordo com o importante para determinado processo, torna possível o cumprimento das obrigações nos prazos pré-determinados. A todo o momento eram identificadas oportunidades de melhoria que poderiam impactar nas responsabilidades de outros membros. Essas oportunidades eram sempre analisadas, documentadas e priorizadas para futura utilização.

É necessário tratar as iniciativas de melhoria como projetos reais, ou seja, a implementação deve ser planejada e monitorada como qualquer projeto de desenvolvimento de software. Inclui-se aí a definição de marcos/pontos de controle e a monitoração constante do progresso, dos custos e dos envolvidos na iniciativa. Isto facilita a percepção das melhorias obtidas a cada etapa (marco) do projeto e serve de incentivo a todos os colaboradores.

A manutenção de uma boa comunicação entre os membros da equipe é fundamental para antecipar riscos, solucionar problemas e aproveitar oportunidades. Devido às restrições de prazo e custo, o impacto de qualquer problema poderia ser alto, mas o aproveitamento de oportunidades poderia potencializar o andamento da iniciativa de melhoria. 


\subsection{Lições Aprendidas na Perspectiva da Instituição Avaliadora}

Algumas lições aprendidas dizem respeito a como se preparar para uma avaliação, outras a cuidados durante a avaliação e outras, ainda, ao que fazer depois de finalizada a avaliação. Estas lições aprendidas são úteis no trabalho como II orientando as empresas em projetos de consultoria para implantação de processos e também no trabalho atuando como IA ao fornecer orientações sobre momentos importantes de todo o processo.

Deve-se começar a preparar a planilha de evidências com bastante antecedência, preferencialmente logo após a implantação da infra-estrutura dos processos. Isso ajudará a identificar possíveis problemas de aderência ao modelo MPS mais cedo.

Deve-se planejar o esforço necessário de preparação da avaliação para aumentar a previsibilidade dos recursos necessários para preparar a organização para a avaliação. Isso inclui, também, a preparação do ambiente para a avaliação com antecedência para evitar problemas que podem afetar a condução da avaliação.

A empresa deve ser preparada para ouvir as críticas dos avaliadores de forma construtiva e evitar criar conflitos devido a divergências na interpretação dos resultados dos processos. Não se devem levar críticas dos avaliadores para o lado pessoal, pois não são as pessoas que estão sendo avaliadas, mas sim a implementação dos processos.

Os colaboradores da empresa a serem entrevistados devem ser orientados para serem mais claros e objetivos possível nas respostas durante as entrevistas. Isto facilita a compreensão do alcance da implementação pela equipe de avaliação.

A empresa deve ser orientada a ter um representante na equipe de avaliação em cada mini-equipe. Com isso, aumenta a segurança da própria empresa de que a implementação realizada foi entendida corretamente pela equipe de avaliação.

Deve-se incorporar os achados da avaliação em um plano de ação, priorizar as ações e gerenciar o plano até todas as ações terem sido concluídas. Se possível, devese, também, disseminar as melhorias identificadas em outras unidades organizacionais que também implementaram os processos, mas não foram avaliadas.

Manter o nível obtido na avaliação não é uma tarefa trivial, exige esforço, determinação da equipe e da alta direção e tem custo alto, mas vale a pena e é imprescindivel para se ter retorno do investimento.

\section{Conclusão e Perspectivas Futuras}

A transposição dos desafios enfrentados permitiu a extração de diversas lições aprendidas na implementação do nível E no MR-MPS na área de Qualidade de Software do LENS. Estas lições aprendidas foram incorporadas pela equipe e serão úteis, tanto nos desenvolvimentos internos e externos da COPPE/UFRJ, como nos trabalhos de consultoria em melhoria de processos de software. É importante destacar, também, uma lição aprendida que, certamente, terá impacto sobre a atuação como avaliadores MPS. A experiência fez com que se percebesse como a avaliação é estressante para a organização que está sendo avaliada. Muito esforço e recursos de todo o tipo estão em jogo neste momento. Ter consciência de que se fez o melhor possível, que a implementação está adequada e que a organização está preparada para a avaliação não elimina o nervosismo e a ansiedade da alta direção e da equipe. Assim sendo, os avaliadores devem lidar com esta realidade com muito cuidado e compreensão. 
Hoje a equipe compreende melhor todos os desafios e limitações que uma organização enfrenta quando decide percorrer o caminho da melhoria contínua de seus processos. Dessa forma, esta experiência foi enriquecedora e auxiliará na busca por formas mais eficazes de implementação em relação a menor custo, menor tempo e mais visibilidade do retorno do investimento nas organizações. Além disso, a COPPE/UFRJ continua os esforços em busca de um ambiente alta maturidade e planejar obter o nível A do MR-MPS até o ano de 2010.

\section{Referências}

Boehm, B. (2006), "A view of 20th and 21st century software engineering", $A C M$, Shanghai, China.

Chrissis, M.B., Konrad, M., Shrum, S. (2006), CMMI (Second Edition): Guidelines for Process Integration and Product Improvement, Addison Wesley Professional.

Ferreira, A.I.F., Santos, G., Cerqueira, R., et al. (2007), "ROI of Software Process Improvement at BL Informática - SPI is Really Worth". In: EuroSPI 2007 - Industrial Proceedings, pp. 12.27 - 12.34, Postdam, Germany, September 2007.

Galotta, C., Zanetti, D., Rocha, A.R. (2004), "Organizational Learning Based on a Customizable Environment for Knowledge Management Using Intranet", v. 2, pp. 2626-2633, Washington, EUA.

ISO/IEC (2003), "Information Technology - Software Process Assessment", Parts 1-9, The International Organization for Standardization and the International Electrotechnical Commission, v. ISO/IEC 15504.

ISO/IEC (2008), "Systems and software engineering - Software life cycle processes", The International Organization for Standardization and the International Electrotechnical Commission, v. ISO/IEC 12207:2008.

Montoni, M., Santos, G., Rocha, A.R., et al. (2006), "Taba workstation: Supporting software process deployment based on CMMI and MR-MPS.BR", v. 4034 NCS, pp. 249-262, Amsterdam, Netherlands.

Osterweil, L.J. (1996), "Improving the quality of software quality determination processes", pp. 90-105, 1996.

Santos, G., Montoni, M., Figueiredo, S., et al. (2007a), "SPI-KM - Lessons Learned from Applying a Software Process Improvement Strategy Supported by Knowledge Management", Product-Focused Software Process Improvement.

Santos, G., Montoni, M., Vasconcellos, J., et al. (2007b), "Implementing Software Process Improvement Initiatives in Small and Medium-Size Enterprises in Brazil". In: Quality of Information and Communications Technology, 2007. QUATIC 2007. 6th International Conference on the, pp. 187-198.

SOFTEX (2007), "MPS.BR: Melhoria de Processo do Software Brasileiro". In: http://www.softex.br/mpsbr/. 